\title{
Study of Age-Related Electrophysiological Changes in Egyptian Eyes
}

\author{
Mona Abdelkader*, Sameh Saleh and Amr M Abdelkader \\ Department of Ophthalmology, University of Mansoura, Egypt
}

Submission: November 11, 2019; Published: January 09, 2020

*Corresponding author: Mona Abdelkader, Department of Ophthalmology, University of Mansoura, Mansoura Ophthalmic Center, Egypt

\begin{abstract}
Purpose: To quantify normal age-related electrophysiological changes in a sample of Mansoura population eyes, Egypt.

Method: One thousand healthy subjects " 2000 eyes " were included in the study. Subjects had been divided into five groups according to age category "10-19 years old, 20-29yrs. old, 30-39yrs. old, 40-49yrs. old and 50-65yrs. old. Each group included 200 subjects. All underwent complete ophthalmological examination, pattern visual evoked potential (P-VEP), flash electroretinogram (ERG), and multifocal ERG. Latency and amplitude of each wave were documented among different age groups.

Results: Aging was found to be associated with statistically significant decrease in the amplitude of flash ERG and similar increase in latency. This is noticed by a significant decrease in the amplitude of rod and cone responses in group 5 compared with group 1 ( $p=0.001)$. Similarly, the delay of latencies of rod and cone in group 5 compared with group 1 was statistically significant $(p<0.009)$ when compared with other groups. In MFERG, there was statistically significant reduction of amplitude with statistically insignificant delay in latencies in all rings with the age. However, p-VEP demonstrate an insignificant age-related change of latencies along different groups.
\end{abstract}

Conclusion: A significant age-related changes in most of the visual electrophysiological parameters must be put in consideration when interpreting these tests

Keywords: Mansoura; Ophthalmological; MFERG; Visual electrophysiological; Electrical; Potential; Vision; Medical ethics; Finger breadth

\section{Introduction}

As the number of the elderly population are increasing, the study of age-related changes in the visual system becomes important. Flash ERG is very precise, noninvasive recording of electrical potential of the retina. It reflects the activity of the entire outer retinal layers. While multifocal ERG is a map function of the central $30^{\circ}$ of retina. Their normal values may differ in variable population [1,2] VEP records the activity of the neuronal circuitry and the cortical responses [3]. Evoked potentials measure the functional status of the sensory systems. Electrophysiological tests results differ according to the way of conduction, so the International Society for Clinical Electrophysiology of Vision (ISCEV) standardized the technique [4,5]. Although, many attempts had tried to standardize ERG responses in variable centers in the world, it is still technically infeasible because of the effect of many factors such as age, sex, refractive error, device type and the operator factor [6-8]. The ISCEV recommends that each center sets up normal values depending on its own equipment and patients. Detection of normal ERG \&VEP parameters are essential to make these tests of valid clinical value [5]. Although different studies have been done on ERG \& VEP worldwide but there were scarce studies from Egypt. The aim of the current study was to detect age effect on ERG \&VEP values.

\section{Subjects and Methods}

This randomized, prospective cross-sectional analytical and hospital-based study was carried out on apparently normal subjects recruited at Mansoura Ophthalmic Center during the period from May 2017 to January 2019. The study included 2000 eyes of 1000 subjects. They were divided into five groups. Group 1: aged from 10 to 19 years old. Group 2 aged from 20 to 29 years old. Group 3 aged from 30 to 39 years old. Group 4 aged from 40 to 49 years. Group 5 aged from 50 to 65 years. The study was carried out in accordance with the tenets of the declaration and approved by Mansoura University medical ethics committee. Each patient signed a written consent. Inclusion criteria included normal subject with normal vision, average intraocular pressure, normal anterior and posterior segments. While exclusion criteria included any history of significant ocular diseases, ocular surgery, error of refraction more than \pm 0.5 and history of neurological, diabetic, heart disease, or neurotropic drugs. All subjects 
underwent complete ophthalmologic examination to exclude any ocular pathology. Electrophysiological tests were recorded using Roland Consult, Brandenburg, Germany system. The room was dimly lit with ambient light. Electrophysiological tests (ERG, VEP) were performed in accordance to ISCEV guidelines [8].

\section{Connection of VEP}

Silver - chloride electrodes of $1 \mathrm{~cm}$ diameter were used for recording. Positive electrode was connected to midline of head two finger breadth above inion (projection at back head). Ground electrode was connected in midline of head at level of ear lobule. Negative electrode was connected to middle of forehead. They were monitored during the procedure through the camera in the monitor. The pattern reversal VEP (P-R VEP) was elicited by a checkerboard like stimulus (check size was 0.15 minute, stimulus frequency: $1.5 \mathrm{~Hz}$, cycle time: 0.66 second, contrast: $97 \%$, square number verticle:20, full field CRT screen stimulus) that reverse in a regular phase frequency( 1 to 2 sweep per second). The subjects were asked to gaze at a red cross in the center of the screen during the recording. Subjects were seated at 1 meter and wearing the reading correction when needed.

\section{Connection of ERG}

Dawson Trick Litskow (DTL) electrode were applied to topically anaesthetized cornea with one ground electrode in the forehead and two temporal reference electrodes.

\section{F-ERG}

After dark adaptation for 20 minutes and pupillary dilation with Mydriacil (tropicamide 1\%) eye drop, The eyes fixate on red light point in the Ganzfeld stimulation globe, then the test was recorded in 5 steps: scotopic rod response (LED white flash stimulus, $25 \mathrm{~dB}, 0.2 \mathrm{~Hz}$, cycle time 5 second, intensity of background off white), scotopic combined response (stimulus intensity : $0 \mathrm{~dB}$, frequency: $0.15 \mathrm{~Hz}$, cycle time 7 second), oscillatory potential (Ops) (intensity: $0 \mathrm{~dB}$, frequency : $0.07 \mathrm{~Hz}$, cycle time 14 second) then light adaptation for 10 minutes then photopic cone response (intensity :0 dB, frequency:0.2 Hz, cycle time: 5 second, background intensity $25 \mathrm{~cd} / \mathrm{m}^{2}$ ) and flicker response recording (intensity :0 dB, frequency: $30 \mathrm{~Hz}$, cycle time 0.035 second, background intensity $25 \mathrm{~cd} / \mathrm{m}^{2}$ ).

\section{MF-ERG}

The stimulus, consisting of (61) hexagons covering a visual field of $30^{\circ}$ was presented on a monitor $30 \mathrm{~cm}$ from the eye. Each hexagon independently alternated between black and white. Subjects fixated at the intersection of the spokes in the center of the stimulus. The recording period was comprised of eight segments of 30 second. Contaminated segments were discarded $\&$ repeated.

\section{Statistical analysis}

Data were analyzed using IBM SPSS software package version 20.0. Qualitative data was presented as median with interquartile range. Quantitative data was represented as mean \pm standard deviation. ANOVA test were used for comparison. One-way ANOVA test was used to compare more than 2 groups of normally quantitative variables with post hoc test to detect within group significance. Difference were considered statistically significant when $\mathrm{P}<0.05$.

\section{Results}

Table 1: Flash ERG among groups in male $(P<0.001)$.

\begin{tabular}{|c|c|c|c|c|c|c|c|c|c|c|c|}
\hline \multirow{2}{*}{ F-ERG } & \multicolumn{2}{|c|}{ Group1 } & \multicolumn{2}{|c|}{ Group2 } & \multicolumn{2}{|c|}{ Group 3} & \multicolumn{2}{|c|}{ Group 4} & \multicolumn{2}{|c|}{ Group 5} & \multirow{2}{*}{$\begin{array}{c}\text { P (Test of } \\
\text { Signifi- } \\
\text { cance) }\end{array}$} \\
\hline & Right & Left & Right & Left & Right & Left & Right & Left & Right & Left & \\
\hline \multicolumn{12}{|c|}{ Scotopic b wave } \\
\hline Amplitude $(\mu v)$ & $100 \pm 23^{\mathrm{A}}$ & $101 \pm 24^{\mathrm{AB}}$ & $110 \pm 29$ & $109 \pm 29$ & $111 \pm 24^{\mathrm{A}}$ & $112 \pm 22^{\mathrm{B}}$ & $95 \pm 27$ & $93 \pm 28^{\mathrm{B}}$ & $88 \pm 27^{\mathrm{ABC}}$ & $87 \pm 22^{\mathrm{ABC}}$ & $\mathrm{P}=0.001$ \\
\hline Latency (ms) & $60 \pm 10^{\text {в }}$ & $59 \pm 11^{\mathrm{AC}}$ & $61 \pm 13^{\mathrm{A}}$ & $60 \pm 13$ & $60 \pm 14^{\mathrm{A}}$ & $61 \pm 14$ & $68 \pm 13^{\mathrm{B}}$ & $69 \pm 15$ & $73 \pm 16^{\mathrm{B}}$ & $72 \pm 14^{\mathrm{AC}}$ & $\mathrm{P}=0.004$ \\
\hline \multicolumn{12}{|c|}{ Combined } \\
\hline $\begin{array}{l}\text { a-wave Ampli- } \\
\text { tude } \mu \mathrm{v}\end{array}$ & $24 \pm 7^{\mathrm{A}}$ & $24 \pm 8^{\mathrm{AB}}$ & $25 \pm 10^{\mathrm{ABC}}$ & $26 \pm 9^{A}$ & $30 \pm 11^{\mathrm{ABC}}$ & $30 \pm 13^{\mathrm{AB}}$ & $21 \pm 14$ & $22 \pm 10^{\mathrm{A}}$ & $20 \pm 12$ & $20 \pm 11$ & $P=0.003$ \\
\hline Latency & $20 \pm 1^{\mathrm{AC}}$ & $20 \pm 2$ & $21 \pm 1$ & $21 \pm 2$ & $23 \pm 4$ & $23 \pm 5$ & $30 \pm 1^{\mathrm{AC}}$ & $31 \pm 4$ & $35 \pm 1$ & $34 \pm 3$ & $\mathrm{P}=0.004$ \\
\hline $\begin{array}{l}\text { b-wave ampli- } \\
\text { tude }\end{array}$ & $280 \pm 40$ & $281 \pm 44^{\mathrm{A}}$ & $295 \pm 50$ & $294 \pm 50$ & $299 \pm 45$ & $298 \pm 49^{A}$ & $270 \pm 42$ & $271 \pm 44$ & $261 \pm 54$ & $25945 \pm$ & $\mathrm{P}=0.002$ \\
\hline Latency & $40 \pm 4^{\text {в }}$ & $38 \pm 4$ & $37 \pm 4$ & $37 \pm 3$ & $37 \pm 4$ & $37 \pm 2$ & $42 \pm 2$ & $43 \pm 2$ & $44 \pm 3^{\text {в }}$ & $44 \pm 3$ & $\mathrm{P}=0.004$ \\
\hline \multicolumn{12}{|c|}{ Oscillatory Potential } \\
\hline Amplitude & $20 \pm 6^{\mathrm{AB}}$ & $19 \pm 9^{\mathrm{A}}$ & $25 \pm 10$ & $26 \pm 9$ & $25 \pm 11^{\mathrm{ABC}}$ & $24 \pm 9^{A}$ & $22 \pm 9$ & $23 \pm 9^{\mathrm{AB}}$ & $20 \pm 9$ & $20 \pm 8$ & $\mathrm{P}=0.004$ \\
\hline Latency & $20 \pm 2^{\text {в }}$ & $20 \pm 3^{A C}$ & $20 \pm 3$ & $20 \pm 2$ & $20.5 \pm 2$ & $20 \pm 1$ & $20 \pm 3^{\text {в }}$ & $20 \pm 3$ & $24 \pm 3$ & $24 \pm 4^{\mathrm{AC}}$ & $\mathrm{P}=0.005$ \\
\hline
\end{tabular}




\begin{tabular}{|c|c|c|c|c|c|c|c|c|c|c|c|}
\hline \multicolumn{12}{|c|}{ Photopic } \\
\hline $\begin{array}{l}\text { a-wave ampli- } \\
\text { tude }\end{array}$ & $29 \pm 8 \mathrm{AB}$ & $29 \pm 7$ & $39 \pm 5$ & $39 \pm 7 \mathrm{ABC}$ & $38 \pm 9$ & $38 \pm 7$ & $30 \pm 9 \mathrm{ABC}$ & $30 \pm 5$ & $28 \pm 9$ АВ & $28 \pm 7$ & $\mathrm{P}=0.001$ \\
\hline Latency & $17 \pm 2^{\text {в }}$ & $17 \pm 2^{\mathrm{A}}$ & $15 \pm 2$ & $15 \pm 1^{\mathrm{AC}}$ & $15 \pm 1$ & $15 \pm 2$ & $19 \pm 2^{\text {в }}$ & $19 \pm 3$ & $20 \pm 3$ & $20 \pm 3 \mathrm{AC}$ & $\mathrm{P}=0.002$ \\
\hline $\begin{array}{l}\text { b-wave Ampli- } \\
\text { tude }\end{array}$ & $62 \pm 20$ & $61 \pm 19^{\mathrm{ABC}}$ & $69 \pm 25$ & $69 \pm 26$ & $68 \pm 23$ & $67 \pm 24^{\mathrm{ABC}}$ & $64 \pm 20$ & $63 \pm 21$ & $63 \pm 22$ & $65 \pm 22$ & $\mathrm{P}=0.003$ \\
\hline b-wave latency & $31 \pm 5^{\text {в }}$ & $31 \pm 6^{\mathrm{AC}}$ & $30 \pm 5^{\mathrm{A}}$ & $30 \pm 4 \mathrm{ABC}$ & $31 \pm 4^{\mathrm{B}}$ & $30 \pm 3$ & $35 \pm 5$ & $36 \pm 5$ & $38 \pm 5^{\mathrm{AC}}$ & $38 \pm 5 \mathrm{ABC}$ & $\mathrm{P}=0.004$ \\
\hline \multicolumn{12}{|c|}{ Flicker } \\
\hline Amplitude & $55 \pm 15^{\mathrm{AB}}$ & $55 \pm 10$ & $60 \pm 10$ & $60 \pm 14^{\mathrm{A}}$ & $63 \pm 13$ & $61 \pm 12$ & $56 \pm 15^{\mathrm{ABC}}$ & $56 \pm 14$ & $51 \pm 11^{\mathrm{AB}}$ & $50 \pm 12$ & $\mathrm{P}=0.002$ \\
\hline Latency & $54 \pm 5^{\text {в }}$ & $55 \pm 4 \mathrm{AC}$ & $50 \pm 3$ & $50 \pm 4$ & $50.5 \pm 5^{\mathrm{ABC}}$ & $50 \pm 2^{\text {в }}$ & $53 \pm 3^{A}$ & $53 \pm 4 \mathrm{AC}$ & $59 \pm 3^{A}$ & $58 \pm 4$ & $\mathrm{P}=0.004$ \\
\hline
\end{tabular}

Table 2: Flash ERG among groups in female.

\begin{tabular}{|c|c|c|c|c|c|c|c|c|c|c|c|}
\hline \multirow{2}{*}{ F-ERG } & \multicolumn{2}{|c|}{ Group 1} & \multicolumn{2}{|c|}{ Group 2} & \multicolumn{2}{|c|}{ Group 3} & \multicolumn{2}{|c|}{ Group 4} & \multicolumn{2}{|c|}{ Group 5} & \multirow{2}{*}{ p } \\
\hline & Right & Left & Right & Left & Right & Left & Right & Left & Right & Left & \\
\hline \multicolumn{12}{|c|}{ Scotopic b wave } \\
\hline Amplitude & $100 \pm 23^{\mathrm{A}}$ & $101 \pm 24$ & $110 \pm 29^{\mathrm{B}}$ & $109 \pm 29$ & $111 \pm 24^{\mathrm{C}}$ & $112 \pm 22$ & $95 \pm 27$ & $93 \pm 28 \mathrm{D}$ & $88 \pm 27^{\mathrm{AB}}$ & $87 \pm 22$ & $\mathrm{P}=0.004$ \\
\hline Latency & $60 \pm 10^{\mathrm{C}}$ & $59 \pm 11^{\mathrm{AB}}$ & $61 \pm 13 \mathrm{ABC}$ & $60 \pm 13$ & $60 \pm 14 \mathrm{ABC}$ & $61 \pm 14$ & $68 \pm 13$ & $69 \pm 15^{c}$ & $73 \pm 16$ & $72 \pm 14^{\mathrm{AB}}$ & $\mathrm{P}=0.003$ \\
\hline \multicolumn{12}{|c|}{ Combined } \\
\hline a-wave Amplitude & $24 \pm 7^{\mathrm{A}}$ & $24 \pm 8^{\mathrm{D}}$ & $25 \pm 10^{\text {в }}$ & $26 \pm 9$ & $30 \pm 11$ & $30 \pm 13$ & $21 \pm 14^{\mathrm{A}}$ & $22 \pm 10$ & $20 \pm 12 \mathrm{D}$ & $20 \pm 11^{\text {B }}$ & $P=0.006$ \\
\hline Latency & $20 \pm 1^{\mathrm{AB}}$ & $20 \pm 2$ & $21 \pm 1$ & $21 \pm 2^{\mathrm{ABC}}$ & $23 \pm 4$ & $23 \pm 5$ & $30 \pm 1$ & $31 \pm 4$ & $35 \pm 1 \mathrm{AB}$ & $34 \pm 3^{\mathrm{ABC}}$ & $\mathrm{P}=0.004$ \\
\hline b-wave amplitude & $280 \pm 40^{\text {B }}$ & $281 \pm 44$ & $295 \pm 50^{A}$ & $294 \pm 50$ & $299 \pm 45$ & $298 \pm 49$ & $270 \pm 42^{\mathrm{B}}$ & $271 \pm 44$ & $261 \pm 54^{\mathrm{A}}$ & $259 \pm 45$ & $\mathrm{P}=0.001$ \\
\hline Latency & $40 \pm 4^{\mathrm{ABC}}$ & $38 \pm 4^{c}$ & $37 \pm 4^{\mathrm{AB}}$ & $37 \pm 3$ & $37 \pm 4^{\mathrm{ABC}}$ & $37 \pm 2$ & $42 \pm 2^{c}$ & $43 \pm 2$ & $44 \pm 3^{\mathrm{AB}}$ & $44 \pm 3$ & $\mathrm{P}=0.007$ \\
\hline \multicolumn{12}{|c|}{ Oscillatory Potential } \\
\hline Amplitude & $20 \pm 6^{\mathrm{A}}$ & $19 \pm 9$ & $25 \pm 10^{\mathrm{D}}$ & $26 \pm 9$ & $25 \pm 11^{\mathrm{A}}$ & $24 \pm 9$ & $22 \pm 9$ & $23 \pm 9$ & $20 \pm 9^{\mathrm{D}}$ & $20 \pm 8$ & $P=0.001$ \\
\hline Latency & $20 \pm 2^{\mathrm{AB}}$ & $20 \pm 3$ & $20 \pm 3 \mathrm{ABC}$ & $20 \pm 2$ & $20.5 \pm 2$ & $20 \pm 1$ & $20 \pm 3$ & $20 \pm 3 \mathrm{ABC}$ & $24 \pm 3 \mathrm{AB}$ & $24 \pm 4$ & $\mathrm{P}=0.009$ \\
\hline \multicolumn{12}{|c|}{ Photopic } \\
\hline a-wave amplitude & $30 \pm 7^{\mathrm{D}}$ & $33 \pm 7$ & $34 \pm 7^{\mathrm{A}}$ & $34 \pm 8$ & $34 \pm 8$ & $34 \pm 8$ & $29 \pm 4^{\mathrm{D}}$ & $29 \pm 7$ & $26 \pm 5^{\mathrm{A}}$ & $26 \pm 7$ & $\mathrm{P}=0.004$ \\
\hline Latency & $17 \pm 2^{\mathrm{c}}$ & $17 \pm 2$ & $15 \pm 2$ & $15 \pm 1$ & $15 \pm 1$ & $15 \pm 2$ & $19 \pm 2$ & $19 \pm 3$ & $20 \pm 3^{c}$ & $20 \pm 3$ & $\mathrm{P}=0.002$ \\
\hline b-wave Amplitude & $62 \pm 20^{\text {B }}$ & $61 \pm 19^{A}$ & $69 \pm 25$ & $69 \pm 26^{\mathrm{B}}$ & $68 \pm 23$ & $67 \pm 24^{\mathrm{A}}$ & $64 \pm 20$ & $63 \pm 21$ & $63 \pm 22^{\mathrm{B}}$ & $65 \pm 22$ & $\mathrm{P}=0.004$ \\
\hline b-wave latency & $31 \pm 5^{\mathrm{ABC}}$ & $31 \pm 6$ & $30 \pm 5 \mathrm{AB}$ & $30 \pm 4$ & $31 \pm 4$ & $30 \pm 3$ & $35 \pm 5$ & $36 \pm 5^{\mathrm{AB}}$ & $38 \pm 5^{\mathrm{ABC}}$ & $38 \pm 5$ & $\mathrm{P}=0.001$ \\
\hline \multicolumn{12}{|c|}{ Flicker } \\
\hline Amplitude & $55 \pm 15^{\mathrm{A}}$ & $55 \pm 10^{\text {B }}$ & $60 \pm 10$ & $60 \pm 14$ & $63 \pm 13$ & $61 \pm 12$ & $56 \pm 15$ & $56 \pm 14$ & $51 \pm 11^{\mathrm{A}}$ & $50 \pm 12$ & $\mathrm{P}=0.004$ \\
\hline Latency & $54 \pm 5^{c}$ & $55 \pm 4{ }^{\mathrm{ABC}}$ & $50 \pm 3$ & $50 \pm 4$ & $50.5 \pm 5$ & $50 \pm 2$ & $53 \pm 3$ & $53 \pm 4$ & $59 \pm 3^{c}$ & $58 \pm 4^{\mathrm{ABC}}$ & $\mathrm{P}=0.005$ \\
\hline
\end{tabular}

Table 3: The amplitude ( $\mu \mathrm{v} / \mathrm{deg} 2)$ of P-wave MF-ERG in male.

\begin{tabular}{|c|c|c|c|c|c|c|c|c|c|c|c|}
\hline \multirow{2}{*}{ MFERG } & \multicolumn{2}{|c|}{ Group 1} & \multicolumn{2}{|c|}{ Group 2} & \multicolumn{2}{|c|}{ Group 3} & \multicolumn{2}{|c|}{ Group 4} & \multicolumn{2}{|c|}{ Group 5} & \multirow{2}{*}{$\mathbf{P}$} \\
\hline & Right & Left & Right & Left & Right & Left & Right & Left & Right & Left & \\
\hline Ring 1 & $71 \pm 13^{\mathrm{A}}$ & $70 \pm 11^{\mathrm{AB}}$ & $80 \pm 10^{\text {в }}$ & $79 \pm 10$ & $81 \pm 11^{\mathrm{c}}$ & $81+10$ & $75+10$ & $75+10$ & $70+9^{c}$ & $70+10^{\mathrm{AB}}$ & 0.005 \\
\hline Ring 2 & $62 \pm 15^{\text {в }}$ & $62 \pm 14$ & $65 \pm 12^{\mathrm{A}}$ & $65 \pm 12$ & $66 \pm 12$ & $66+12^{\mathrm{AB}}$ & $60+14$ & $60+14^{A}$ & $56+13^{\mathrm{B}}$ & $55+12^{\mathrm{AB}}$ & 0.006 \\
\hline Ring 3 & $52 \pm 9 \mathrm{AB}$ & $52 \pm 9$ & $55 \pm 10^{\mathrm{ABC}}$ & $55 \pm 11$ & $57 \pm 10$ & $57+10$ & $51+9$ & $52 \pm 9$ & $50 \pm 10^{\mathrm{AB}}$ & $50 \pm 11$ & 0.008 \\
\hline Ring 4 & $41 \pm 11^{\mathrm{A}}$ & $41 \pm 11$ & $45 \pm 9$ & $46 \pm 10$ & $46 \pm 10$ & $46 \pm 11$ & $42 \pm 8$ & $42 \pm 8$ & $40 \pm 9^{A}$ & $40 \pm 10$ & 0.005 \\
\hline Ring 5 & $32 \pm 9^{c}$ & $31 \pm 8$ & $37 \pm 10$ & $37 \pm 11$ & $38 \pm 9$ & $38 \pm 11$ & $33 \pm 11$ & $33 \pm 11$ & $30 \pm 7^{c}$ & $30 \pm 7$ & 0.003 \\
\hline Sup-nasal quadrant & $30 \pm 7^{\mathrm{AC}}$ & $30 \pm 8$ & $35 \pm 9 \mathrm{AD}$ & $35 \pm 9$ & $36 \pm 9$ & $36 \pm 9 \mathrm{ABC}$ & $29 \pm 10$ & $29 \pm 10$ & $25 \pm 9 \mathrm{AC}$ & $25 \pm 9$ & 0.001 \\
\hline
\end{tabular}


JOJ Ophthalmology

\begin{tabular}{|c|c|c|c|c|c|c|c|c|c|c|c|}
\hline $\begin{array}{c}\text { Sup-temporal } \\
\text { quadrant }\end{array}$ & $30 \pm 11^{\mathrm{ABC}}$ & $31 \pm 10^{\mathrm{AD}}$ & $37 \pm 9$ & $37 \pm 9$ & $37 \pm 8$ & $37 \pm 8$ & $30 \pm 9^{\mathrm{ABC}}$ & $30 \pm 10$ & $26 \pm 10^{\mathrm{AD}}$ & $26 \pm 9$ & 0.009 \\
\hline Inf-nasal quadrant & $29 \pm 10^{\mathrm{AD}}$ & $29 \pm 10^{\mathrm{AC}}$ & $36 \pm 11$ & $37 \pm 10^{\mathrm{ABC}}$ & $37 \pm 9$ & $37 \pm 9$ & $29 \pm 10^{\mathrm{AD}}$ & $29 \pm 10^{\mathrm{ABC}}$ & $26 \pm 6$ & $27 \pm 8 \mathrm{AC}$ & 0.007 \\
\hline $\begin{array}{c}\text { Infero-temporal } \\
\text { quadrant }\end{array}$ & $30 \pm 11^{\mathrm{AC}}$ & $30 \pm 9 \mathrm{ABC}$ & $38 \pm 8$ & $39 \pm 8$ & $39 \pm 8$ & $39 \pm 7$ & $30 \pm 9$ & $30 \pm 10$ & $27 \pm 7^{\mathrm{AC}}$ & $27 \pm 7 \mathrm{ABC}$ & 0.005 \\
\hline
\end{tabular}

Table 4: Amplitude (( $\mu \mathrm{v} / \mathrm{deg} 2)$ of $\mathrm{p} \_$wave of MFERG in female.

\begin{tabular}{|c|c|c|c|c|c|c|c|c|c|c|c|}
\hline \multirow{2}{*}{ MFERG } & \multicolumn{2}{|c|}{ Group 1} & \multicolumn{2}{|c|}{ Group 2} & \multicolumn{2}{|c|}{ Group 3} & \multicolumn{2}{|c|}{ Group 4} & \multicolumn{2}{|c|}{ Group 5} & \multirow{2}{*}{$\mathbf{P}$} \\
\hline & Right & Left & Right & Left & Right & Left & Right & Left & Right & Left & \\
\hline Ring 1 & $7 \mathrm{o} \pm 13$ & $70 \pm 11$ & $80 \pm 10$ & $79 \pm 10$ & $81 \pm 11$ & $81 \pm 10$ & $74 \pm 10$ & $75+11$ & $70 \pm 9$ & $70 \pm 10$ & 0.003 \\
\hline Ring 2 & $60 \pm 15$ & $62 \pm 14$ & $64 \pm 12$ & $65 \pm 12$ & $65 \pm 12$ & $65 \pm 12$ & $60+14$ & $60+14$ & $56+13$ & $56 \pm 12$ & 0.005 \\
\hline Ring 3 & $52 \pm 9$ & $52 \pm 9$ & $55 \pm 10$ & $55 \pm 11$ & $57 \pm 10$ & $56 \pm 10$ & $51+9$ & $52 \pm 9$ & $50 \pm 10$ & $50 \pm 11$ & 0.001 \\
\hline Ring 4 & $41 \pm 11$ & $41 \pm 11$ & $45 \pm 9$ & $46 \pm 10$ & $46 \pm 10$ & $46 \pm 11$ & $42 \pm 8$ & $42 \pm 8$ & $40 \pm 9$ & $40 \pm 10$ & 0.003 \\
\hline Ring 5 & $32 \pm 9$ & $31 \pm 8$ & $37 \pm 10$ & $37 \pm 11$ & $38 \pm 9$ & $38 \pm 11$ & $33 \pm 11$ & $33 \pm 11$ & $30 \pm 7$ & $30 \pm 7$ & 0.006 \\
\hline Sup-nasal quadrant & $30 \pm 7$ & $30 \pm 8$ & $35 \pm 9$ & $35 \pm 9$ & $36 \pm 9$ & $36 \pm 9$ & $29 \pm 10$ & $29 \pm 10$ & $25 \pm 9$ & $24 \pm 9$ & 0.003 \\
\hline Sup-temporal quadrant & $30 \pm 11$ & $31 \pm 10$ & $36 \pm 9$ & $37 \pm 9$ & $37 \pm 8$ & $37 \pm 8$ & $30 \pm 9$ & $30 \pm 10$ & $26 \pm 10$ & $26 \pm 9$ & 0.009 \\
\hline Inf-nasal quadrant & $29 \pm 10$ & $29 \pm 10$ & $36 \pm 11$ & $37 \pm 10$ & $37 \pm 9$ & $37 \pm 9$ & $29 \pm 10$ & $29 \pm 10$ & $26 \pm 6$ & $27 \pm 8$ & 0.008 \\
\hline Infero-temporal quadrant & $30 \pm 11$ & $30 \pm 9$ & $38 \pm 8$ & $39 \pm 8$ & $39 \pm 8$ & $39 \pm 7$ & $30 \pm 9$ & $30 \pm 10$ & $27 \pm 7$ & $26 \pm 7$ & 0.001 \\
\hline
\end{tabular}

Table 5: Latency (ms) of $p$-wave of MFERG ( $p>0.1)$.

\begin{tabular}{|c|c|c|c|c|c|c|c|c|c|c|c|}
\hline \multirow{2}{*}{ MFERG } & \multicolumn{2}{|c|}{ Group 1} & \multicolumn{2}{|c|}{ Group 2} & \multicolumn{2}{|c|}{ Group 3} & \multicolumn{2}{|c|}{ Group 4} & \multicolumn{2}{|c|}{ Group 5} & \multirow{2}{*}{$\mathbf{P}$} \\
\hline & Right & Left & Right & Left & Right & Left & Right & Left & Right & Left & \\
\hline Ring 1 & $49 \pm 2$ & $49 \pm 1$ & $50 \pm 3$ & $502 \pm$ & $50 \pm 2$ & $50 \pm 3$ & $51 \pm 3$ & $52 \pm 3$ & $52 \pm 2$ & $52 \pm 3$ & 0.9 \\
\hline Ring2 & $48 \pm 3$ & $48 \pm 2$ & $49 \pm 2$ & $49 \pm 2$ & $49 \pm 3$ & $49 \pm 2$ & $51 \pm 2$ & $51 \pm 2$ & $51 \pm 2$ & $51 \pm 2$ & 0.467 \\
\hline Ring3 & $49 \pm 3$ & $48 \pm 3$ & $49 \pm 3$ & $49 \pm 4$ & $49 \pm 3$ & $49 \pm 2$ & $50 \pm 3$ & $50 \pm 2$ & $50 \pm 3$ & $50 \pm 4$ & 0.87 \\
\hline Ring4 & $49 \pm 3$ & $49 \pm 2$ & $49 \pm 2$ & $49 \pm 2$ & $50 \pm 2$ & $50 \pm 3$ & $50 \pm 3$ & $50 \pm 2$ & $50 \pm 3$ & $50 \pm 3$ & 0.22 \\
\hline Ring5 & $49 \pm 2$ & $49 \pm 4$ & $50 \pm 3$ & $50 \pm 3$ & $50 \pm 4$ & $50 \pm 3$ & $50 \pm 2$ & $50 \pm 3$ & $51 \pm 4$ & $51 \pm 3$ & 0.1 \\
\hline
\end{tabular}

Table 6: P-VEP among groups.

\begin{tabular}{|c|c|c|c|c|c|c|c|c|c|c|c|}
\hline \multirow{2}{*}{$\mathbf{P}$} & \multicolumn{2}{|c|}{ Group 5} & \multicolumn{2}{|c|}{ Group 4} & \multicolumn{2}{|c|}{ Group 3} & \multicolumn{2}{|c|}{ Group 2} & \multicolumn{2}{|c|}{ Group 1} & \multirow{2}{*}{ p-VEP } \\
\hline & Left & Right & Left & Right & Left & Right & Left & Right & Left & Right & \\
\hline$<0.008$ & $9 \pm 9$ & $9 \pm 8$ & $8 \pm 8$ & $8 \pm 8$ & $11 \pm 8$ & $11 \pm 9$ & $12 \pm 10$ & $12 \pm 10$ & $10 \pm 6$ & $9 \pm 5$ & P100 Amplitude( $\mu v)$ \\
\hline$>0.5$ & $95.5 \pm 9$ & $96 \pm 9$ & $96.8 \pm 4$ & $97 \pm 4$ & $96 \pm 3$ & $96 \pm 3$ & $96 \pm 4$ & $95 \pm 3$ & $95 \pm 2$ & $95 \pm 3$ & P100 Latency(ms) \\
\hline
\end{tabular}

A total of 1000 subjects (2000 eyes), 550 female (55\%) and 450 males (45\%) were included in the study. The study was divided into five groups; Group 1: comprised of two hundred normal subjects (100 female, 100 male) aged from 10 to 19 years old. Group 2: comprised of two hundred subjects(110 female, 90 male) aged from 20 to 29 years old Group 3: comprised of two hundred subjects (120 female, 80 male) aged from 30 to 39 years old .Group 4: comprised of 200 subjects (120 female, 80 male) aged from 40 to 49 years. Group 5: comprised of 200 subjects ( 100 females, 100 male) aged from 50 to 65 years. As regards F-ERG; maximum amplitudes were in group 2 and minimal amplitudes were in group 5 while the latencies were maximum in group 5 and minimum in group1. There was a tendency to decrease in amplitudes and increase in latencies with the age; (Table 1,2 \& Figure 1,2). There was an overall significant difference in between all groups especially between the group 1 and group $5(\mathrm{p}=0.001)$. In MF-ERG, there was a statistically significant reduction in amplitudes with age. But there was an insignificant increase in latencies with age (Table 3-5 \& Figure 3). In P-VEP, there was a statistically significant reduction in amplitude, however latencies expressed statistically insignificant increase with age (Table 6 \& Figure 4-6). There was insignificant difference between right and left eye ( $p>0.1$ ) in the overall visual electrophysiological tests, similarly, there was insignificant difference between female and male $(p>0.5)$. 

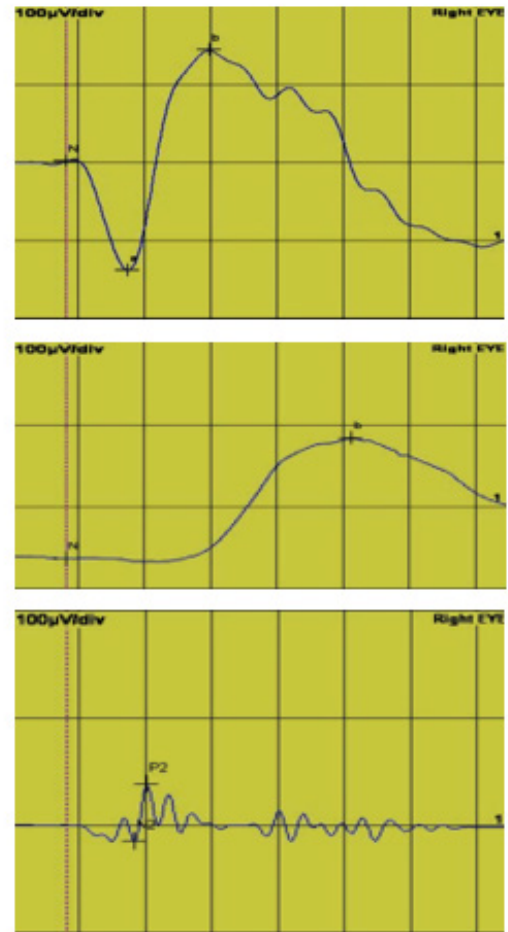

Figure 1: Normal Scotopic F-ERG (there are normal of amplitude of scotopic rod, combined rod \&cone responses and normal oscillatory potential).
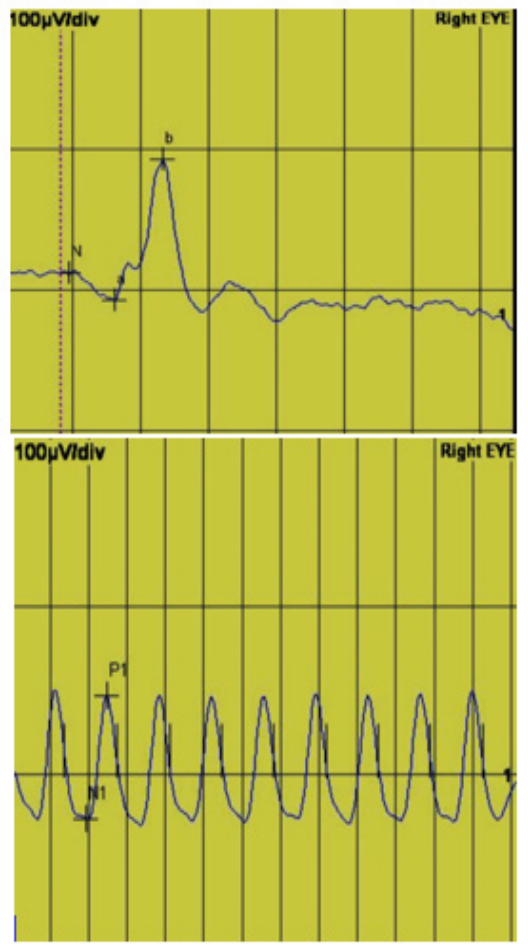

Figure 2: Normal Photopic F-ERG (It shows normal cone amplitude and latency and normal oscillatory potential). 


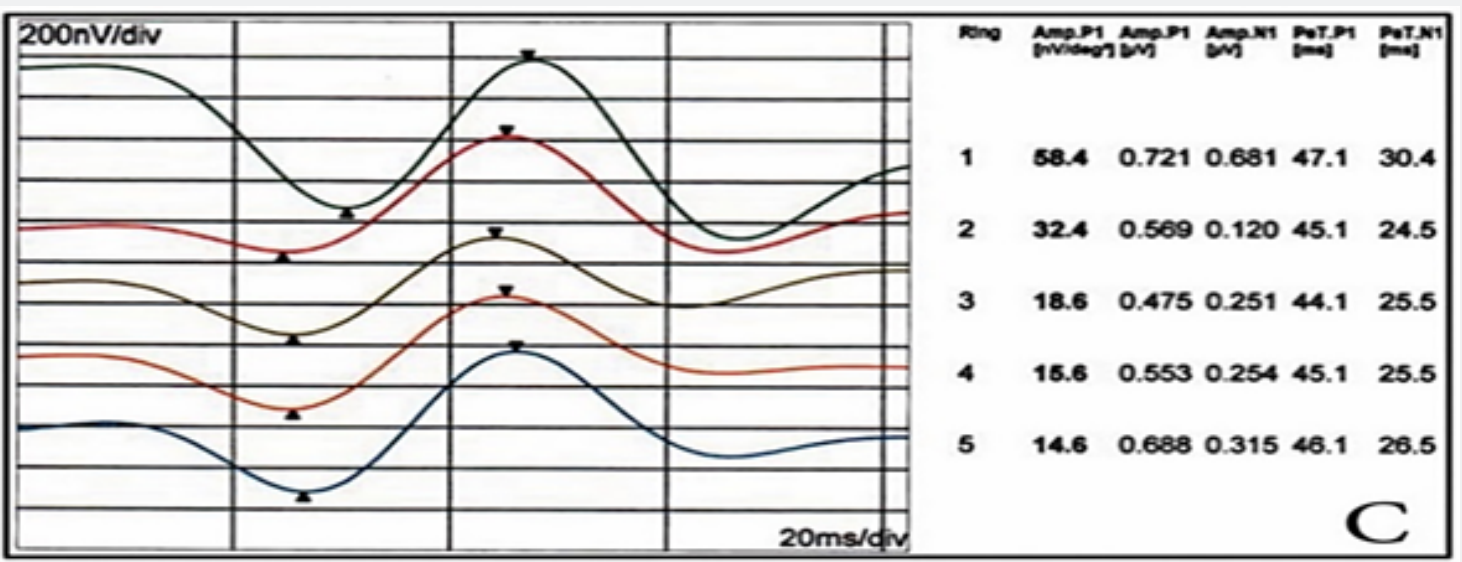

Figure 3: Normal MFERG (there are normal latencies and amplitudes in all rings)..

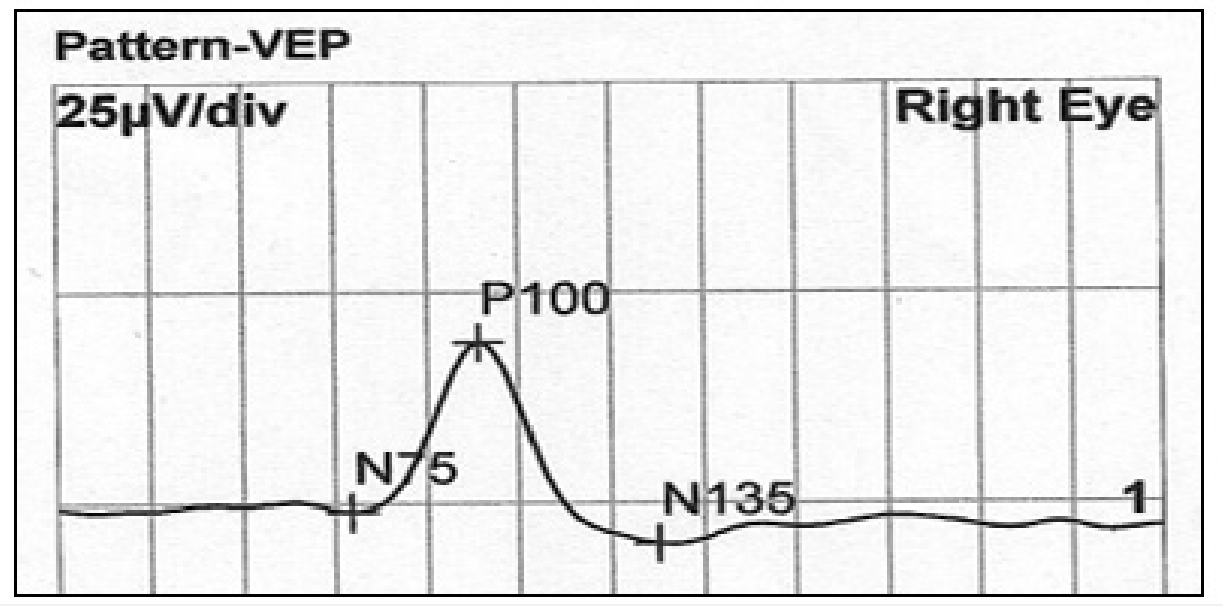

Figure 4: Normal P-VEP in both right and left (It shows normal latencies and amplitudes of N75, P100, N135).

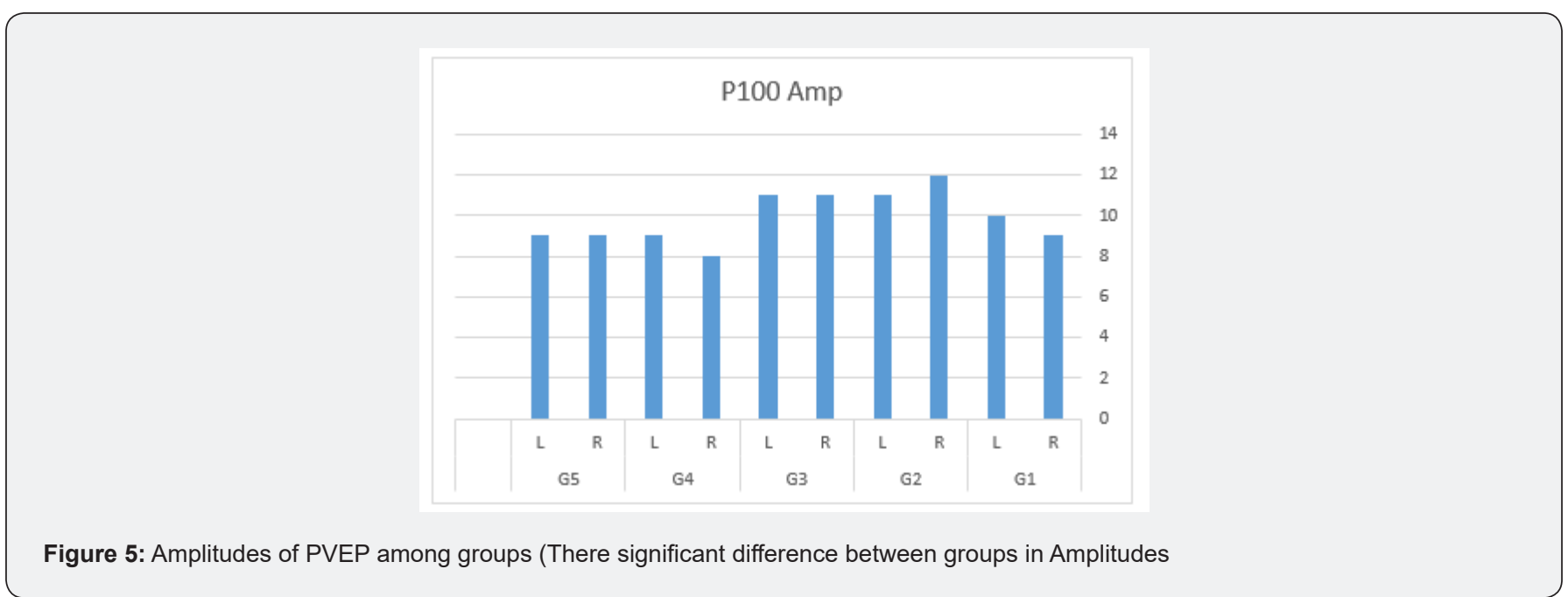




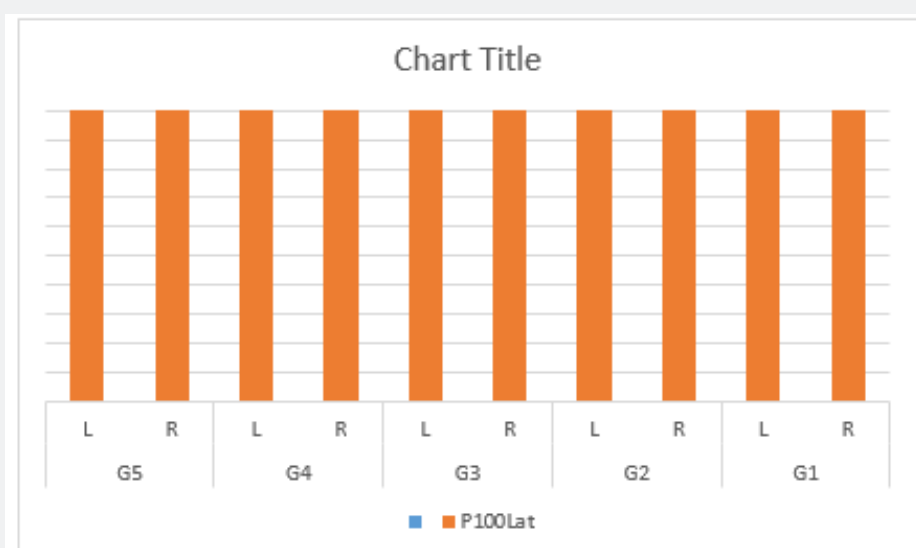

Figure 6: latencies of P-VEP in both right and left (There was insignificant difference between groups in latencies).

\section{Discussion}

Detection of normative and age-related changes in ERG and VEP parameters is essential to make those tests of a real clinical usefulness. There are different factors affecting the values obtained from electrophysiological recording, consequently it is difficult to establish a fixed normal range. These factors include race, gender, age, pupillary size, error of refraction, duration and intensity of light stimuli, dark/light adaptation period, electrode type and site and others $[9,10]$. So, it is important that each center establishes its own normative parameters to avoid misinterpretation of results. This study assesses normal ranges for ERG \& VEP responses in different age groups in Mansoura Population in Egypt. All procedures were done according ISCV [8]. This study included 2000 eyes divided into five groups. There was reduction of amplitudes in all five steps (scotopic $24 \mathrm{~dB}$, scotopic $0 \mathrm{~dB}$, oscillatory potential, photopic and flicker) of F-ERG in group 5 compared with other groups $(\mathrm{p}<0.001)$. The values of rod and cone response were reduced with age. The causes of reduction in responses of F-ERG with ageing were claimed to be due to reduction in photopigment optical density, and bipolar or Muller cell death related to retinal ageing [11].

Similarly [11], found that there was an increase in ERG latency values in dark and light adapted in old subjects $>50$ years. However, [11] observed no significant change in oscillatory potential with age. Also, found a similar reduction in all F-ERG values with old age $[12,13]$. The average results of F-ERG parameters (Table $1 \& 2$ ) in this study were lower than those of the studies conducted [14-16] found that the average amplitude of scotopic was $149 \pm 144 \mu \mathrm{V}$, of photopic was $129 \pm 86 \mu \mathrm{V}, 30-\mathrm{Hz}$ flicker was $134 \pm 38 \mu \mathrm{V}$, Oscillatory potential was $25.6 \pm 13.9 \mu \mathrm{V}$, latency time of scotopic was $0.53 .5 \pm 29 \mathrm{~ms}$, latency time photopic was $22.9 \pm 7.7 \mathrm{~ms}$, and flicker was $54.3 \pm 7.5 \mathrm{~ms}$.The causes of lower values in the current study may be racial or technical factors such as electrode placement or pupil size. A statistically significant effect of gender on ERG recordings has been reported in previous studies $[8,10,11]$. In India, females precede males in the age-related changes. In contrast, western males reflect greater aging changes than females [16]. However, we did not observe any significant difference between male and female subjects in this study. Similarly, [12,13] reported no gender difference in their studies. Similarly, in this study, there was no statistically significant difference between right and left eyes. ( $p>0.5)$.

F-ERG determines the functional integrity of the retina (rods and cones in the outer retina \& associated pathways in the middle and inner layers of the retina), While MF-ERG is a topographic map of retinal function over central 30 degree. The current study found a significant age-related reduction in amplitude of MF-ERG. However, there was an insignificant age-related change in implicit time (Table 3,4 \& Figure 3). Other studies of MF-ERG produced inconstant results found a reduction of amplitude without change in latency time [14]. Another study found both statistically significant reduction of amplitude and a delay in latency time with age. [15-17] reported an increase in implicit time with age. The reasons of different results between studies might be attributed to different (age range, subjects' number, study design or pupil size). There were varying causes for age related reduction of amplitude including intracellular aberrancies in the inner segments of cone photoreceptors, mitochondrial abnormalities in the foveal cones [18], reduction of foveal cone pigment density and age related decreased number of retinal pigment epithelial cells, cone and rod photoreceptors, and ganglion cells [19]. In this study, VEP was found to have significant age-related reduction in the amplitude of p wave and an insignificant increase in latency. Similarly, [20] found a reduction in P100 with little variations in latency with age. The aging differences may be due to; environmental factors like exposure to solar radiation that leads to excitation toxicity of visual cell which results in accelerated apoptosis and death 
of neurons) [7] dietary factors like deficiency of Vitamin A that activates photo-transduction which leads to lower calcium concentration causing death of rods and neurons) [21], and genetic factors as genes like neuronal Rac-1 and rdy that increase oxidation stress and damage. Also, this still could be explained by changes in ocular media that lead to reductions in illuminance of the visual stimulus, senile changes in neurons and the cumulative exposure to high energy photons from solar radiation [22]. The presented data were performed under strict controlled conditions and can be used as a native reference values and valid as data base for worldwide meta-analysis.

\section{References}

1. Bouskila J, Javadi P, Palmour RM, Bouchard JF, Ptito M (2014) Standardized Full-Field Electroretinography in the Green Monkey 9(10): e111569.

2. Good KL, Kass PH, Park SA, Nestorowicz N, Ofri R (2013) Effects of chemical restraint on electroretinograms recorded sequentially in awake, sedated, and anesthetized dogs. Am J Vet Res 74(7): 1036-1042.

3. Onofrj M, Thomas A, Iacono D, Andreamatteo G, Paci C (2001) Agerelated changes of evoked potentials. Clin Neurophysiol 31(2): 83-103.

4. Odom JV, Bach M, Brigell M (2016) ISCEV standard for clinical visual evoked potentials (2016 update). Doc Ophthalmol 133(1): 1-9.

5. Marmor MF, Holder GE, Seeliger MW, Yamamoto S (2004) Standard for clinical electroretinography Doc Ophthalmol 108(2): 107-114.

6. Organisciak DT, Vaughan DK (2010) Retinal light damage: Mechanisms and protection. Prog Retin Eye Res 29(2): 113-134.

7. Fain GL (2006) Why photoreceptors die (and why they don't). Oessays 28(4): 344-354.

8. Mc Culloch DL, Marmor MF, Brigell MG, Hamilton R, Holder G, et al. (2015) ISCEV Standard for full-field clinical electroretinography. Doc Ophthalmol 130(1): 1-12.

9. Ryan SJ, Hinton DR, Harbert GS (2013) Retina. ( $5^{\text {th }}$ edn), Publisher: Elsevier.

10. Mosavat M (2010) Electrophisiology of the Eye and Fundus Diseases. $\left(1^{\text {st }}\right.$ edn), Publisher: Sahel Andisheh Tehran.
11. Corîci AC, Alexandru DO, Corîci OM1, Puianu M, Iancău M, et al. (2015) Variability of Normal Values of Electroretinogram Parameters Due to Aging in Healthy Individuals. Current Health Sciences Journal 41(1): 29-34. July

12. Parvaresh M, Ghiasian L, Falavarjani, GK, Sanjari MS (2009) Normal Values of Standard Full Field Electroretinography in an Iranian Population. J Ophthalmic Vis Res 4(2): 97-101.

13. Shokouhian M, Alizadeh Y, Khosravi B, Jafarzadehpour E, Souri H (2017) Normal Values of Standard Full Field Electroretinography in Healthy Subjects in Northern Iran. Caspian J Neurol Sci 3(3): 135-142.

14. Seiple W, Thasarat S, Janet P, Clemens C, Holopigian K, et al. (2003) Multifocal Electroretinography as a Function of Age: The Importance of Normative Values for Older Adults. Invest Ophthalmol Vis Sci 44(4): 1783-1792.

15. Jackson RG, Ortega JDL, Girkin C, Rosenstiel CE, Owsley C (2002) Aging-related changes in the multifocal electroretinogram. J Opt Soc Am A 19(1): 185-189.

16. Gerth C, Garcia SM, Ma L, Keltner JL, Werner JS (2002) Multifocal electroretinogram: age-related changes for different luminance levels. Graefes Arch Clin Exp Ophthalmol 240(3): 202-208.

17. Fortune B, Johnson CA (2002) The decline of photopic multifocal electroretinogram responses with age is primarily due to pre-retinal optical factors. J Opt Soc Am A 19(1): 173-184.

18. Barron MJ, Johnson MA, Andrew RM (2001) Mitochondrial abnormalities in aging macular photoreceptors. Invest Ophthalmol Vis Sci 42(12): 3016-3022.

19. Curcio CA (2001) Photoreceptor topography in ageing and age-related maculopathy. Eye 15(3): 376-383.

20. Phurailatpam J, Sharma AK, Singh R (2014) Effect of age on patternreversal visual evoked potentials in Indian population, Int J Clin Exp Physiol 1(2): 152-156.

21. Van Norren D, Gorgels TG (2011) The action spectrum of photochemical damage to the retina: A review of monochromeatic threshold data. Photochem Photobiol 87(4): 747-753.

22. Haruta M, Bush RA, Kjellstrom S, Vijyaysarathy C, Zeng Y, et al. (2009) Depleting Rac-1 in mouse rod photoreceptors protects them from photo-oxidative stress without affecting their structure or function. $\mathrm{Pr}$ Natl Acad Sci USA 106(23): 9397-9402.

\begin{tabular}{|l|}
\hline \multicolumn{1}{|c|}{ Your next submission with Juniper Publishers } \\
will reach you the below assets \\
- Quality Editorial service \\
- Swift Peer Review \\
- Reprints availability \\
- E-prints Service \\
- Manuscript Podcast for convenient understanding \\
- Global attainment for your research \\
- Manuscript accessibility in different formats \\
( Pdf, E-pub, Full Text, Audio) \\
- Unceasing customer service \\
Track the below URL for one-step submission \\
https://juniperpublishers.com/online-submission.php
\end{tabular}

